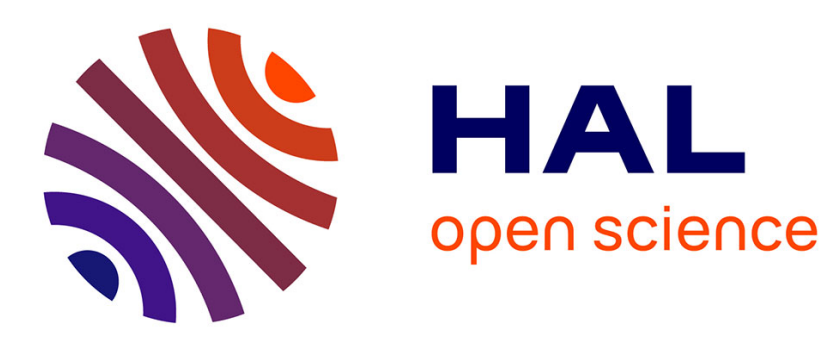

\title{
Convective flow field measurement by speckle velocimetry
}

R. Meynart

\section{To cite this version:}

R. Meynart. Convective flow field measurement by speckle velocimetry. Revue de Physique Appliquée, 1982, 17 (5), pp.301-305. 10.1051/rphysap:01982001705030100 . jpa-00245000

\section{HAL Id: jpa-00245000 https://hal.science/jpa-00245000}

Submitted on 1 Jan 1982

HAL is a multi-disciplinary open access archive for the deposit and dissemination of scientific research documents, whether they are published or not. The documents may come from teaching and research institutions in France or abroad, or from public or private research centers.
L'archive ouverte pluridisciplinaire HAL, est destinée au dépôt et à la diffusion de documents scientifiques de niveau recherche, publiés ou non, émanant des établissements d'enseignement et de recherche français ou étrangers, des laboratoires publics ou privés. 
Classification

Physics Abstracts

$6.30 \mathrm{G}-42.30-47.80$

\title{
Convective flow field measurement by speckle velocimetry
}

\author{
R. Meynart \\ Université Libre de Bruxelles, Service des Milieux Continus, 87, av. Ad. Buyl, B-1050 Bruxelles, Belgique
}

(Reç le 7 décembre 1981, révisé le 2 février 1982, accepté le 8 février 1982)

\begin{abstract}
Résumé. - Le champ de vitesse au sein d'une cellule de Rayletgh-Bénard cylindrique est déterminé au moyen d'une technique dérivée de la photographie de speckle. L'analyse des photographies s'effectue automatiquement par deux méthodes différentes : (i) Le filtrage du spectre de fréquences spatiales permet d'obtenir des franges d'égale composante de vitesse; (ii) L'éclairement localisé de la photographie par un faisceau laser produit des franges de Young dont le pas est déterminé au moyen d'un système d'analyse d'image. De nouvelles techniques sont proposées pour l'analyse numérique de ces franges de Young.
\end{abstract}

\begin{abstract}
A 2-D velocity map in a cylindrical Rayleigh-Bénard flow is determined by means of a speckle photography technique. Automatic velocity measurements are performed by two different methods : (i) Isovelocity contours are drawn by spatial filtering; (ii) Local illumination by a laser beam produces Young's fringes which are analysed by an image processing system. New methods are proposed for digital analysis of the speckle fringes.
\end{abstract}

1. Introduction. - Techniques for local velocity measurements are well developped in experimental fluid mechanics and commercially available instruments (hot wire velocimeters, laser Doppler velocimeters) provide accurate measures of the time dependence of local flow velocity. However, methods for measuring instantaneous velocity fields are less developped. Photographic techniques are attractive, but they prove quite time consuming for velocity measurements. Over the last few years, methods have been used for speckle photographs analysis in solid mechanics which permit quantitative interpretation of multiple exposure photographs of fluid flows [1].

Two analysis techniques are available. Isovelocity contours can be obtained by filtering the spatial frequency spectrum of the photograph. Point-bypoint measurements of the local velocity can be performed by analysis of Young's fringes produced by local illumination.

This paper describes the application of speckle photography to Rayleigh-Bénard convection in a cylindrical cell. Local velocity information is obtained by a digital image processing system. Given the properties of the speckle noise present in the fringes, new methods are proposed for automatic digital processing of the fringes.

2. Experimental set-up. - The Rayleigh-Bénard cell (shown in Fig. 1) consists of two cylindrical copper discs (diameter : $50 \mathrm{~mm}$ ) separated by a glass cylinder which constitutes the lateral wall of the cell. The aspect ratio (diameter versus height) is 2.0 . This assembly is contained in a parallelipipedic box whose lateral walls are made of glass plates. Both the inside and the outside of the inner cylinder are filled with glycerol. The match-

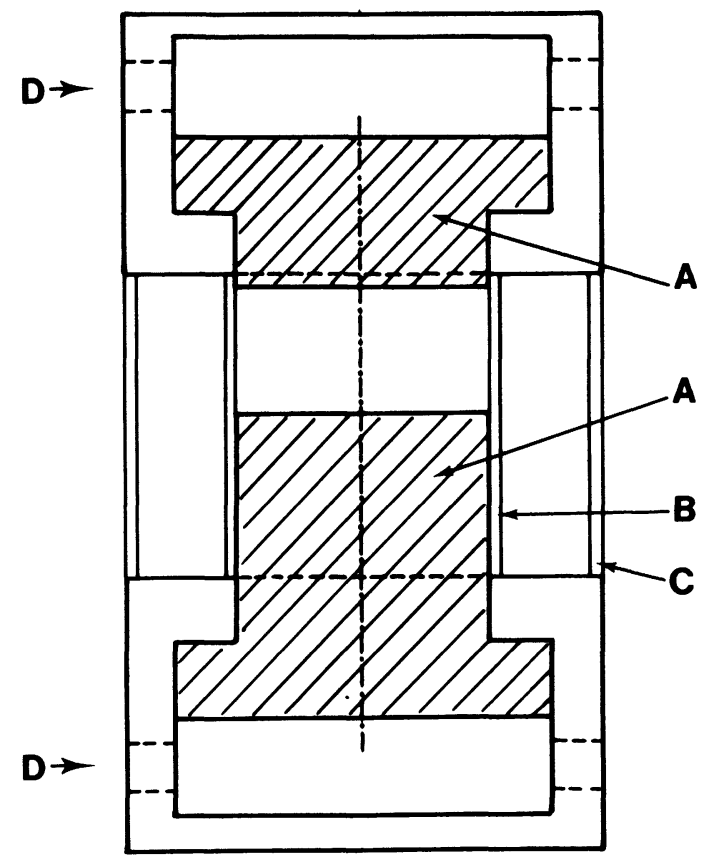

Fig. 1. - Section through the axis of the convection cell. (A); Copper plates. (B); Inner glass cylinder. (C); Glass plates. (D); Water inlets. 
ing between the refractive indices of glycerol and glass prevents dioptric effects which would introduce severe aberrations. The temperature difference between the two horizontal copper surfaces is regulated by temperature-controlled water flows. In this geometry, the flow pattern that arises at the critical Rayleigh number consists of one roll of cylindrical symmetry [2]. This convection torus remains steady for moderate temperature differences (in our case, $\left.\Delta T / \Delta T_{\mathrm{c}}=1.66\right)$.

The beam from an argon laser $(1.7 \mathrm{~W}$ at $514.5 \mathrm{~nm})$ is focused in a vertical plane containing the cell axis. A mechanical chopper produces 10 pulses (each of $20 \mathrm{~ms}$ duration) separated by $3-\mathrm{s}$ intervals. The beam illuminates the diffusing particles (with $5.8 \mu \mathrm{m}$ diameter) suspended in the fluid contained in the inner cylinder. The picture obtained from a camera focused on the illuminated plane is similar to those obtained in conventional tracer methods (see Fig. 2a). The main difference between speckle flow velocimetry

a)

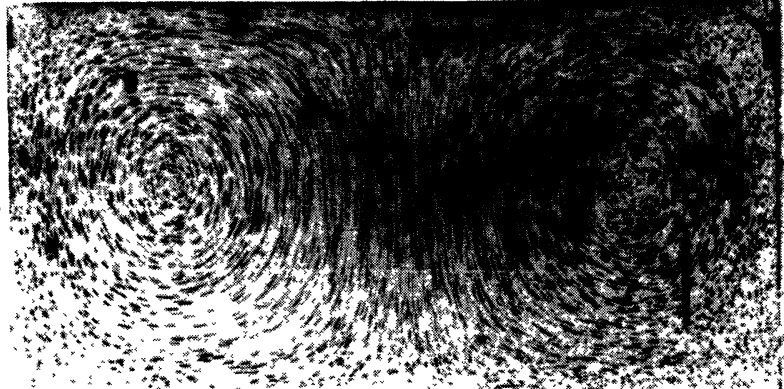

b)

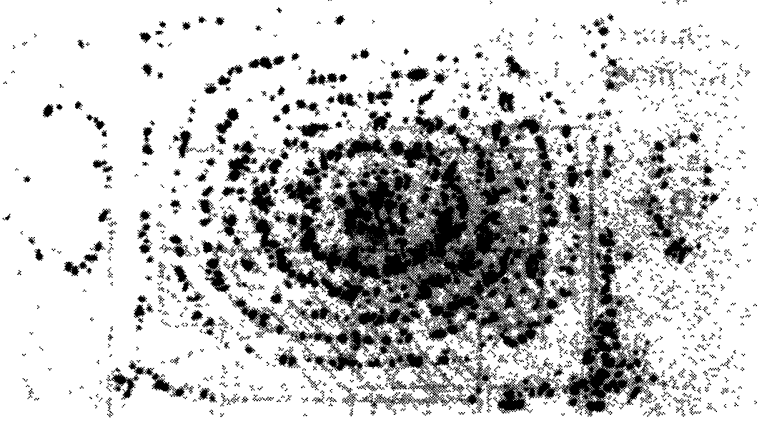

c)

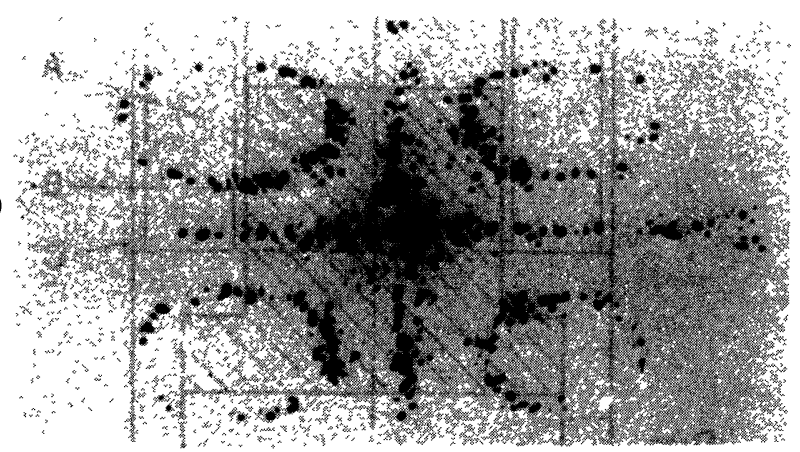

Fig. 2. - (a); Multiple exposure photograph of the convection flow. $(b)$; Filtered image (the fringes are recorded with reversed contrast), vertical velocity component, $\Delta v_{z}=15.5 \mu \mathrm{m} / \mathrm{s}$. (c); Filtered image, horizontal velocity component, $\Delta v_{x}=18.8 \mu \mathrm{m} / \mathrm{s}$. and speckle photography in solid mechanics is the absence of a real speckle in the image plane.

It should be noted that an incoherent light source could be used, however laser light provides high power densities in a well-defined and thin plane. Note also that, due to the gaussian profile of the focal line, the intensity of the light diffused by the particles can vary strongly, inducing non-uniformity in the shapes of the images of the particles. However, it is observed that this effect does not affect the final results. Therefore particle monodispersity is not a crucial factor in this experiment.

3. Velocity measurement by spatial filtering. Processing the photograph with a conventional spatial filter (Fig. 3) provides directly a velocity map of the 2-D flow pattern. This method is fast and convenient.

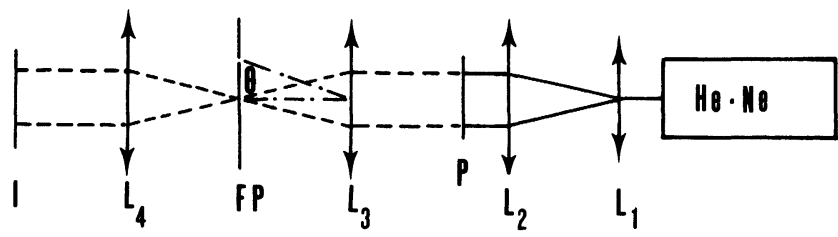

Fig. 3. - Spatial filter. $L_{1}, L_{2}$ : beam expander; $P$ : Photograph ; FP : Fourier plane with the mask $; \mathrm{L}_{3}, \mathrm{~L}_{4}$ : Transform lenses; I : Image plane.

The transmittance of the photograph is given by

$$
T(\mathbf{r})=\sum_{i=1}^{N} f_{i}(\mathbf{r}) *\left\{\sum_{k=0}^{n-1} \delta\left[\mathbf{r}-\left(\mathbf{r}_{i}+k \mathbf{d}\right)\right]\right\}
$$

where $\mathbf{r}$ is the position vector, * means a $2-D$ convolution, $n=$ number of exposures, $N=$ number of particles, $\mathbf{r}_{i}=$ initial position of the $i$-th particle, $\mathbf{d}=$ displacement of the particles, and $f_{i}(\mathbf{r})$ is the function describing the shape of the image of the $i$-th particle.

In the Fourier plane (FP in Fig. 3), the amplitude is expressed in the form :

$$
U\left(\mathbf{r}_{\mathrm{f}}\right)=\operatorname{TF}[T(\mathbf{r})] P\left(\mathbf{r}_{\mathrm{f}}\right)
$$

where $\mathbf{r}_{\mathrm{f}}$ is the position vector in the Fourier plane, TF means a $2-D$ Fourier transform and $P\left(r_{f}\right)$ is the transmittance of the filter.

If the filter is a circular hole (with radius $a$ ), whose center is located at $\mathbf{r}_{0}$, corresponding to a spatial frequency $\mathbf{f}_{0}=\mathbf{r}_{0} / \lambda f$, the intensity in the image plane ( $I$ in Fig. 3) may be expressed by

$$
\begin{array}{r}
I\left(\mathbf{r}_{\mathbf{p}}\right)=C \mid T\left(-\mathbf{r}_{\mathbf{p}}\right) *\left[\exp \left(-2 \pi j \mathbf{r}_{\mathbf{p}} \mathbf{f}_{0}\right) \times\right. \\
\left.\times \frac{J_{1}\left(2 \pi r_{p} a / \lambda f\right)}{\left(2 \pi r_{p} a / \lambda f\right)}\right]\left.\right|^{2}
\end{array}
$$


where $\mathbf{r}_{\mathrm{p}}$ is the position vector in the image plane, $C$ is a constant, $\lambda$ is the wavelength and $f$ is the focal length of the transform lenses.

The convolution in equation (2) will be extremum in the regions where the projection $d_{\mathrm{p}}$ of the displacement on the direction defined by the filtering hole is a multiple of the period of the oscillatory term $\exp \left(-2 \pi j \mathbf{r}_{\mathrm{p}} \mathbf{f}_{0}\right)$. The projected displacement $d_{\mathrm{p}}$ obeys the relation $d_{\mathrm{p}}=n \lambda / \theta$, where $n$ is an integer and $\theta$ the angle shown in figure 3 . Thus isovelocity contours are drawn by the spatial filter as illustrated in Figures $2 b$ and $2 c$. The interferences between independent particles contained in the area defined by the factor $J_{1}\left(2 \pi r_{\mathrm{p}} a / \lambda f\right) /\left(2 \pi r_{\mathrm{p}} a / \lambda f\right)$ in equation (2) produce the speckle present in the filtered image.

It must be pointed out that the quality of the isovelocity fringes is essentially a result of the multiple exposure technique. The multiple beam interferences cause the same sharpening and increase in brightness of the isovelocity fringes as in a Fabry-Pérot interferometer.

The isovelocity maps in figure 2 allow a rapid whole-field examination of the features of the flow pattern. For instance one observes that the vertical velocity in the vicinity of the cell axis is much larger than near the cell wall. This is quite obvious because the particles descending near the wall must ascend near the axis and therefore because of mass conservation, the velocity must be larger in the vicinity of the center of the cell.

4. Velocity measurement by digital acquisition and processing of Young's fringes. - 4.1 AcQuisition OF THE FRINGES. - Instead of filtering the whole photograph to obtain isovelocity contours, illuminating a small portion of the photograph for local velocity measurement provides Young's fringes in the back focal plane of a converging lens [1]. To overcome the inconvenients of visual processing, the image is recorded by an acquisition system; a block diagram is given in figure 4. Rotation of the mirror followed by displacement of the photodiode in a plane parallel

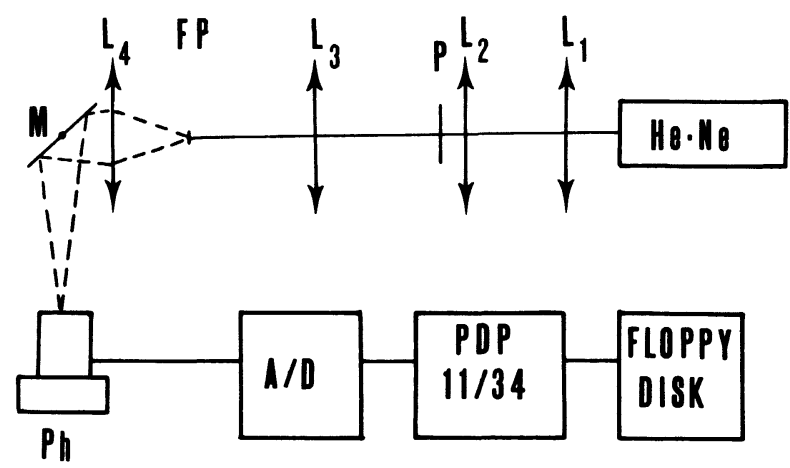

Fig. 4. - Image acquisition system (top view). $\mathrm{L}_{1}, \mathrm{~L}_{2}$ : Lenses for beam size adjustment; $\mathbf{P}$ : Photograph; $\mathbf{L}_{3}$ : Transform lens ; FP : Fourier plane with stop for undiffracted beam ; $\mathrm{L}_{4}$ : Relay lens ; $\mathrm{M}$ : Rotating mirror ; $\mathrm{Ph}$ : Photodiode mounted on a motorized table. to the axis of the rotating mirror define a system of 128 lines and 128 columns equivalent to those of a digitizing camera. The main advantage of this set-up is the good $S / N$ ratio (resulting in 12-bit digitization) obtained by selection of an appropriate photodiode. A digitizing camera would be much faster but the $S / N$ ratio would be poorer. It should be noted that a rather low number of pixels is sufficient to extract the periodicity information.

Due to the convolutive form of equation (1), it follows that the intensity recorded by the acquisition system will be :

$$
I(m, n)=A(m, n) B(m, n) \quad 0 \leqslant m, n \leqslant 127
$$

where $B(m, n)$ is periodic along an unknown direction in the $(m, n)$ plane and $A(m, n)$ is the speckle diffraction halo. The purpose of the processing system is to extract the periodicity information from $B(m, n)$ by suppressing the noise contained in $A(m, n)$.

4.2 Method 1 : IMAge PROCESSING BY 1-D AVERAGING. - Recently, digital processing systems have been proposed for speckle photography in solid mechanics $[3,4,5,6]$. In these methods, a mechanical (or electronic) rotation aligns the fringes parallel to the columns of the acquisition system. In this case, $B(m, n)$ depends only on the $m$-index and averaging of the lines yields :

$f(m)=\sum_{n=0}^{127} I(m, n)=B(m) \sum_{n} A(m, n)=B(m) A^{\prime}(m)$.

The period of $f(m)$ (Figs. $5 a$ and $6 a$ ) can be determined

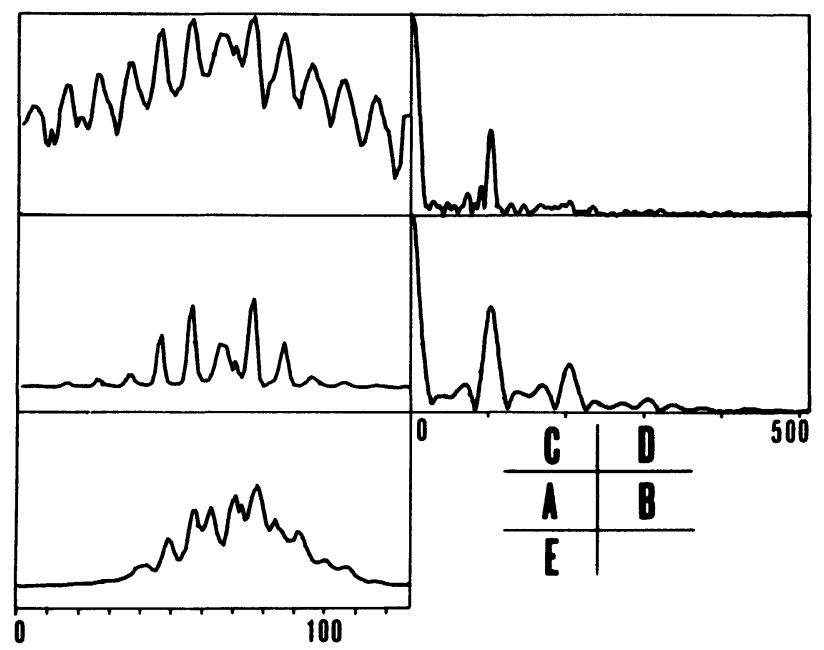

Fig. 5. - Typical exemple of processed image (moderate fringe density). (a); Intensity integrated parallel to the fringes : $f(m)=\sum_{n} I(m, n)$. (b); $\mid$ FFT $f(m) \mid(512$ points from 1024 points FFT). $(c) ; \log f(m) .(d) ; \mid$ FFT $[\log f(m)] \mid$. $(e)$; Intensity integrated perpendicular to the fringes : $\sum I(m, n)$, note the noisy aspect of the curve due to the presence of speckle in $I(m, n)$ (vertical scales : arbitrary units ; horizontal axes : column number $(a, c)$; channel number $(b, d)$; line number $(e))$. 
by Fourier analysis. By computing the 1-D discrete Fourier transform by a FFT algorithm, we have :

$$
\mid \text { FFT } f(m)|=| \text { FFT } B(m) * \text { FFT } A^{\prime}(m) \mid
$$

there $*$ denotes the $1-D$ discrete convolution.

For very low particle displacements (very low fringe densities), the Fourier peaks are deformed because FFT $A^{\prime}(m)$ is broader than the separation of the peaks (see Fig. 6). In this case, the apparent maxima of the Fourier peaks do not yield a reliable measure of the particle displacement. In solid mechanics, a second integration after $90^{\circ}$ rotation gives $A^{\prime}(m)$; the latter is introduced into $(3)$ to recover $B(m)$ which is a constant amplitude periodic function. The resolution of the

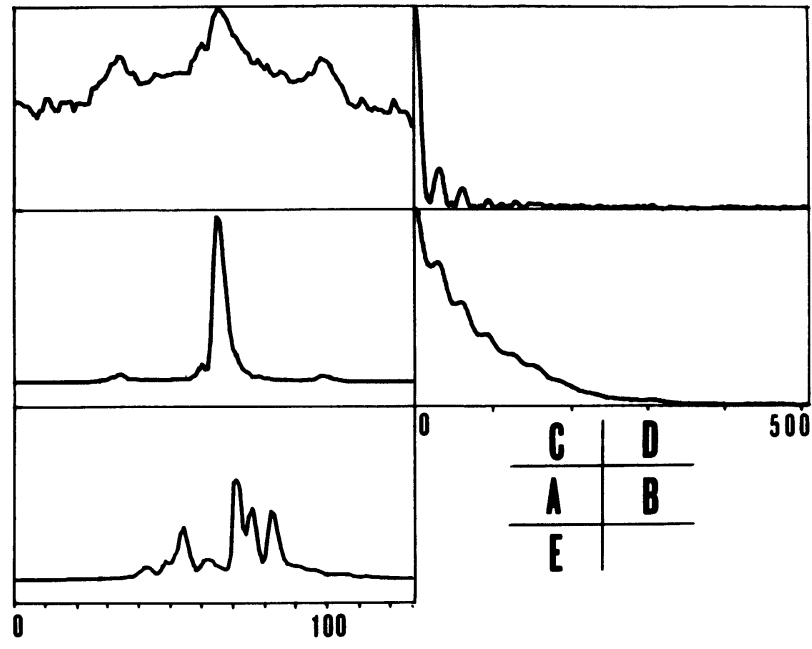

Fig. 6. - Typical exemple of processed image (very low fringe density). See caption of figure 5 .

peaks of $\mid$ FFT $B(m) \mid$ is improved because the width of these peaks is minimized [6]. The difficulty in obtaining a smooth $A^{\prime}(m)$ curve (Figs. $5 e$ and $6 e$ ) is probably due to the non-gaussian statistics of the speckle. There is no speckle in the classical sense, but a composite diffraction pattern which results of interferences between the very small number of particles located in the illuminated region. However, recovering a constant amplitude signal is still possible by using a method closely related to the "cepstrum " technique known in acoustics [7].

Since the frequency spectrum of $\log A^{\prime}(m)$ is concentrated near the origin, by computing

$\mid$ FFT $[\log f(m)] \mid=$

$$
=\mid \text { FFT }[\log B(m)]+\text { FFT }\left[\log A^{\prime}(m)\right] \mid \text {, }
$$

we obtain narrower and better resolved peaks (Fig. 6d). In practice, a constant (of the order of $10^{-4}$ of the maximum value of $f(m))$ must be added to $f(m)$ in order to eliminate the computational singularity of $\log$ zero.

Comparison of the data so obtained shows that both methods give the same results (within $1 \%$ ) if at least two diffraction orders are present in the field. Thus it can be inferred that five fringes in the speckle halo represent the limit for which direct Fourier analysis of $f(m)$ yields accurate results. Below this limit, the " logarithm " method can still give a measure of the velocity. However, the logarithm operation decreases the $S / N$ ratio and the spectrum becomes noisy (Figs. $5 d$ and $6 d$ ).

For the convection experiment, a sequence of measurements has been performed along an horizontal line containing the two zero-velocity points (see Fig. $2 a$ ). Because the velocity is always vertical along this line, the preceding methods are applicable with our acquisition set-up. The curve in figure 7 gives the velocity profile determined by the "logarithm " method. This curve can be compared with the velocity maps (Figs $2 b$ and $2 c$ ) and shows the same features of the spatial profile.

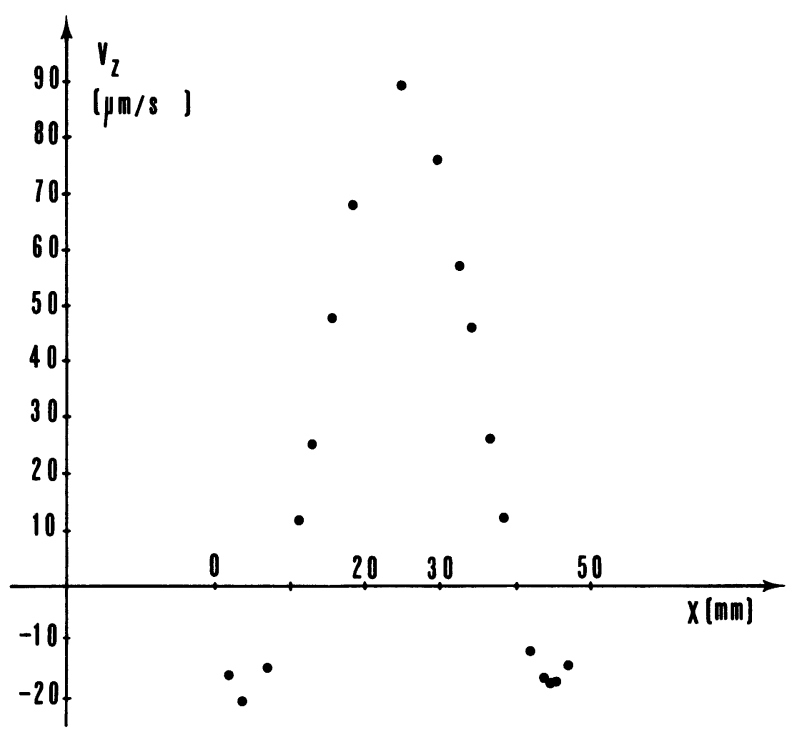

Fig. 7. - Vertical velocity measured by the "logarithm » method along an horizontal line containing the two zerovelocity points.

\subsection{MeTHOD 2 : IMAGE PROCESSING BY AN AVERAGED} AUTOCORRELATION TECHNIQUE. - Rotation of the fringes for alignment is a drawback of the preceding methods. A different technique for velocity component determination may be proposed. With arbitrary orientation of the fringes, direct averaging of the line intensities cannot yield a periodic signal. However, averaging over the autocorrelations of each line gives a periodic signal whose frequency is proportional to the projection of the velocity onto the lines of the acquisition system. The velocity component along the $m$-axis can be obtained by computing

$$
\begin{aligned}
& g(u)=\sum_{n}\left\{\frac{\sum_{m=0}^{127} I(m, n) I(m+u, n)}{\sum_{m=0}^{127}[I(m, n)]^{2}}\right\} \\
& -127 \leqslant u \leqslant 127 .
\end{aligned}
$$


Normalization of the autocorrelations gives an equivalent weight to each line whereas, without this operation, $g(u)$ is strongly biased by the small number of lines where the intensity is large (near the center of the image). On the other hand, by virtue of equation (4), the lines where the intensity is too low are discarded from the sum over $n$ and so autocorrelations from lines where only noise is present are eliminated.

As in the methods described above, measurement of the period is performed by Fourier analysis. Since $g(u)$ is an average over autocorrelations, the discrete Fourier transform FFT $g(u)$ is a real and even function. It should be pointed out that, for computer time saving, FFT $g(u)$ can be calculated by averaging over the squared Fourier transforms of each line.

Using this method, the convective velocity has been mesured for the same points as described above. Comparison shows that the values computed by the autocorrelation method agree within $1 \%$ with the evaluations from the "logarithm " technique when at least two diffraction orders are present in the speckle halo. Figure 8 illustrates this method for the same fringes as in figure 5 .

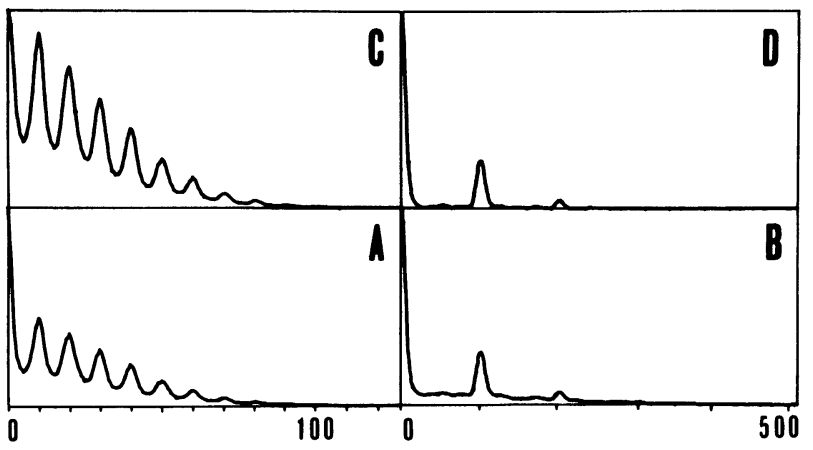

Fig. 8. - Same image as in figure 5 processed by the autocorrelation method. (a); Averaged autocorrelation $g(u)$. (b); FFT $g(u) .(c) ; g^{\prime}(u)=g(u) /\left[1+\exp \left(-u^{2} / p^{2}\right)\right]$. (d); $\mid$ FFT $g^{\prime}(u) \mid$ (vertical scales : a.u.).

A further improvement is shown in figures $8 c$ and $8 d$. The peak around $u=0$ in $g(u)$ is mainly due to the speckle present in the diffraction halo. This peak, responsible for high-frequency components in the
Fourier transform is squeezed by computing the function

$$
g^{\prime}(u)=\frac{g(u)}{1+\exp \left(-u^{2} / p^{2}\right)}
$$

where $p$ is an adjustable parameter. The resolution of the peaks is improved by this empirical correction.

The autocorrelation method is attractive because it yields velocity components for arbitrarily oriented fringes. An orthogonal velocity component can be determined by inverting $m$ and $n$ in equation (4). Thus automation of the method is feasible. This method is more computer time consuming, but simple specialized processors would compute autocorrelations very fast. Moreover, using a digitizing camera instead of the slow and complex rotating mirror system would speed up the acquisition process. An inconvenient of the autocorrelation method is the lower limit for fringe densities which however is not very severe (at least two diffraction orders).

5. Conclusion. - The different techniques for speckle photograph processing show that accurate and rapid instruments can be realized for 2- $D$ velocity measurements. Spatial frequency filtering is an inherently automatic method and digital processing of the Young's fringes enables rapid determination of the local velocity. The two new methods, the "logarithm " technique for very low fringe densities applications and the autocorrelation technique for automatic velocity determination, show that accurate processing systems can be designed which are well suited for fluid flow measurements. The application to very low velocities presented in this paper is only one example of the capabilities of the speckle method. The technique should be applicable to high velocities as well. Current research is performed with pulsed lasers in order to investigate such possibilities.

Acknowledgments. - Support by the Fonds National de la Recherche Scientifique (F.N.R.S.) is gratefully acknowledged. The author would like to thank the Von Karman Institute for Fluid Dynamics (VKI) for useful collaboration in this research.

\section{References}

[1] Meynart, R., Appl. Opt. 19 (1980) 1385.

[2] STORK, K. and MÜLLER, U., J. Fluid Mech. 71 (1975) 231.

[3] Maddux, G. E., Moorman, S. L. and Corwin, R. R., Air Force Flight Dynamics Laboratory Report AFFDL-TM-78-109-FBE (1978).

[4] Kreitlow, H. and KreIs, T., Proc. SPIE 210 (1980) 18.
[5] Ineichen, B., Eglin, P. and Dandliker, R., Appl. Opt. 19 (1980) 2191.

[6] Kaufmann, G. H., Ennos, A. E., Gale, B. and Pugh, D. J., J. Phys. E 13 (1980) 579.

[7] OPPENHEIM, A. and SCHAFER, R., Digital signal processing (Prentice-Hall, New York) 1975, Chapter 10. 